\title{
Some Special Matrices of Real Elements and Their Properties
}

\author{
Xiquan Liang \\ Qingdao University of Science \\ and Technology \\ China
}

\author{
Fuguo Ge \\ Qingdao University of Science \\ and Technology \\ China
}

\author{
Xiaopeng Yue \\ Qingdao University of Science \\ and Technology \\ China
}

Summary. This article describes definitions of positive matrix, negative matrix, nonpositive matrix, nonnegative matrix, nonzero matrix, module matrix of real elements and their main properties, and we also give the basic inequalities in matrices of real elements.

MML identifier: MATRIX10, version: 7.8 .03 4.75.958

The terminology and notation used here are introduced in the following articles: [2], [9], [3], [12], [1], [5], [8], [4], [7], [11], [6], and [10].

\section{Some Special Matrices of Real Elements}

We use the following convention: $a, b$ are elements of $\mathbb{R}, i, j, n$ are natural numbers, and $M, M_{1}, M_{2}, M_{3}, M_{4}$ are matrices over $\mathbb{R}$ of dimension $n$.

Let $M$ be a matrix over $\mathbb{R}$. We say that $M$ is positive if and only if:

(Def. 1) For all $i, j$ such that $\langle i, j\rangle \in$ the indices of $M$ holds $M_{i, j}>0$.

We say that $M$ is negative if and only if:

(Def. 2) For all $i, j$ such that $\langle i, j\rangle \in$ the indices of $M$ holds $M_{i, j}<0$.

We say that $M$ is nonpositive if and only if: 
(Def. 3) For all $i, j$ such that $\langle i, j\rangle \in$ the indices of $M$ holds $M_{i, j} \leq 0$.

We say that $M$ is nonnegative if and only if:

(Def. 4) For all $i, j$ such that $\langle i, j\rangle \in$ the indices of $M$ holds $M_{i, j} \geq 0$.

Let $M_{1}, M_{2}$ be matrices over $\mathbb{R}$. The predicate $M_{1} \sqsubseteq M_{2}$ is defined as follows:

(Def. 5) For all $i, j$ such that $\langle i, j\rangle \in$ the indices of $M_{1}$ holds $\left(M_{1}\right)_{i, j}<\left(M_{2}\right)_{i, j}$. We say that $M_{1}$ is less or equal with $M_{2}$ if and only if:

(Def. 6) For all $i, j$ such that $\langle i, j\rangle \in$ the indices of $M_{1}$ holds $\left(M_{1}\right)_{i, j} \leq\left(M_{2}\right)_{i, j}$.

Let $M$ be a matrix over $\mathbb{R}$. The functor $|: M:|$ yielding a matrix over $\mathbb{R}$ is defined by:

(Def. 7) len $|: M:|=$ len $M$ and width|:M:| = width $M$ and for all $i, j$ such that $\langle i$, $j\rangle \in$ the indices of $M$ holds $|: M:|_{i, j}=\left|M_{i, j}\right|$.

Let us consider $n$ and let us consider $M$. Then $-M$ is a matrix over $\mathbb{R}$ of dimension $n$.

Let us consider $n$ and let us consider $M_{1}, M_{2}$. Then $M_{1}+M_{2}$ is a matrix over $\mathbb{R}$ of dimension $n$.

Let us consider $n$ and let us consider $M_{1}, M_{2}$. Then $M_{1}-M_{2}$ is a matrix over $\mathbb{R}$ of dimension $n$.

Let us consider $n$, let $a$ be an element of $\mathbb{R}$, and let us consider $M$. Then $a \cdot M$ is a matrix over $\mathbb{R}$ of dimension $n$.

Let us observe that there exists a matrix over $\mathbb{R}$ which is positive and nonnegative and there exists a matrix over $\mathbb{R}$ which is negative and nonpositive.

Let $M$ be a positive matrix over $\mathbb{R}$. One can check that $M^{\mathrm{T}}$ is positive.

Let $M$ be a negative matrix over $\mathbb{R}$. Note that $M^{\mathrm{T}}$ is negative.

Let $M$ be a nonpositive matrix over $\mathbb{R}$. One can verify that $M^{\mathrm{T}}$ is nonpositive.

Let $M$ be a nonnegative matrix over $\mathbb{R}$. Observe that $M^{\mathrm{T}}$ is nonnegative.

Let us consider $n$. Observe that $\left(\begin{array}{ccc}1 & \ldots & 1 \\ \vdots & \ddots & \vdots \\ 1 & \ldots & 1\end{array}\right)^{n \times n}$ is positive and nonnegative and $\left(\begin{array}{ccc}-1 & \ldots & -1 \\ \vdots & \ddots & \vdots \\ -1 & \ldots & -1\end{array}\right)^{n \times n} \quad$ is negative and nonpositive.

Let us consider $n$. One can verify that there exists a matrix over $\mathbb{R}$ of dimension $n$ which is positive and nonnegative and there exists a matrix over $\mathbb{R}$ of dimension $n$ which is negative and nonpositive.

We now state a number of propositions:

(1) For every element $x_{1}$ of $\mathbb{R}_{\mathrm{F}}$ and for every real number $x_{2}$ such that $x_{1}=x_{2}$ holds $-x_{1}=-x_{2}$. 
(2) For every matrix $M$ over $\mathbb{R}$ such that $\langle i, j\rangle \in$ the indices of $M$ holds $(-M)_{i, j}=-M_{i, j}$

(3) For all matrices $M_{1}, M_{2}$ over $\mathbb{R}$ such that $\operatorname{len} M_{1}=\operatorname{len} M_{2}$ and width $M_{1}=$ width $M_{2}$ and $\langle i, j\rangle \in$ the indices of $M_{1}$ holds $\left(M_{1}-M_{2}\right)_{i, j}=$ $\left(M_{1}\right)_{i, j}-\left(M_{2}\right)_{i, j}$.

(4) For every matrix $M$ over $\mathbb{R}$ such that len $(a \cdot M)=\operatorname{len} M$ and $\operatorname{width}(a$. $M)=$ width $M$ and $\langle i, j\rangle \in$ the indices of $M$ holds $(a \cdot M)_{i, j}=a \cdot M_{i, j}$.

(5) The indices of $M=$ the indices of $|: M:|$.

(6) $|: a \cdot M:|=|a| \cdot|: M:|$.

(7) If $M$ is negative, then $-M$ is positive.

(8) If $M_{1}$ is positive and $M_{2}$ is positive, then $M_{1}+M_{2}$ is positive.

(9) If $-M_{2} \sqsubseteq M_{1}$, then $M_{1}+M_{2}$ is positive.

(10) If $M_{1}$ is nonnegative and $M_{2}$ is positive, then $M_{1}+M_{2}$ is positive.

(11) If $M_{1}$ is positive and $M_{2}$ is negative and |: $M_{2}:|\sqsubseteq|: M_{1}: \mid$, then $M_{1}+M_{2}$ is positive.

(12) If $M_{1}$ is positive and $M_{2}$ is negative, then $M_{1}-M_{2}$ is positive.

(13) If $M_{2} \sqsubseteq M_{1}$, then $M_{1}-M_{2}$ is positive.

(14) If $a>0$ and $M$ is positive, then $a \cdot M$ is positive.

(15) If $a<0$ and $M$ is negative, then $a \cdot M$ is positive.

(16) If $M$ is positive, then $-M$ is negative.

(17) If $M_{1}$ is negative and $M_{2}$ is negative, then $M_{1}+M_{2}$ is negative.

(18) If $M_{1} \sqsubseteq-M_{2}$, then $M_{1}+M_{2}$ is negative.

(19) If $M_{1}$ is positive and $M_{2}$ is negative and $\left|: M_{1}:\right| \sqsubseteq\left|: M_{2}:\right|$, then $M_{1}+M_{2}$ is negative.

(20) If $M_{1} \sqsubseteq M_{2}$, then $M_{1}-M_{2}$ is negative.

(21) If $M_{1}$ is positive and $M_{2}$ is negative, then $M_{2}-M_{1}$ is negative.

(22) If $a<0$ and $M$ is positive, then $a \cdot M$ is negative.

(23) If $a>0$ and $M$ is negative, then $a \cdot M$ is negative.

(24) If $M$ is nonnegative, then $-M$ is nonpositive.

(25) If $M$ is negative, then $M$ is nonpositive.

(26) If $M_{1}$ is nonpositive and $M_{2}$ is nonpositive, then $M_{1}+M_{2}$ is nonpositive.

(27) If $M_{1}$ is less or equal with $-M_{2}$, then $M_{1}+M_{2}$ is nonpositive.

(28) If $M_{1}$ is less or equal with $M_{2}$, then $M_{1}-M_{2}$ is nonpositive.

(29) If $a \leq 0$ and $M$ is positive, then $a \cdot M$ is nonpositive.

(30) If $a \geq 0$ and $M$ is negative, then $a \cdot M$ is nonpositive.

(31) If $a \geq 0$ and $M$ is nonpositive, then $a \cdot M$ is nonpositive.

(32) If $a \leq 0$ and $M$ is nonnegative, then $a \cdot M$ is nonpositive. 
(33) $|: M:|$ is nonnegative.

(34) If $M_{1}$ is positive, then $M_{1}$ is nonnegative.

(35) If $M$ is nonpositive, then $-M$ is nonnegative.

(36) If $M_{1}$ is nonnegative and $M_{2}$ is nonnegative, then $M_{1}+M_{2}$ is nonnegative.

(37) If $-M_{1}$ is less or equal with $M_{2}$, then $M_{1}+M_{2}$ is nonnegative.

(38) If $M_{2}$ is less or equal with $M_{1}$, then $M_{1}-M_{2}$ is nonnegative.

(39) If $a \geq 0$ and $M$ is positive, then $a \cdot M$ is nonnegative.

(40) If $a \leq 0$ and $M$ is negative, then $a \cdot M$ is nonnegative.

(41) If $a \leq 0$ and $M$ is nonpositive, then $a \cdot M$ is nonnegative.

(42) If $a \geq 0$ and $M$ is nonnegative, then $a \cdot M$ is nonnegative.

(43) If $a \geq 0$ and $b \geq 0$ and $M_{1}$ is nonnegative and $M_{2}$ is nonnegative, then $a \cdot M_{1}+b \cdot M_{2}$ is nonnegative.

\section{Some Basic Inequalities in Matrices of Real Elements}

Next we state a number of propositions:

(44) If $M_{1} \sqsubseteq M_{2}$, then $M_{1}$ is less or equal with $M_{2}$.

(45) If $M_{1} \sqsubseteq M_{2}$ and $M_{2} \sqsubseteq M_{3}$, then $M_{1} \sqsubseteq M_{3}$.

(46) If $M_{1} \sqsubseteq M_{2}$ and $M_{3} \sqsubseteq M_{4}$, then $M_{1}+M_{3} \sqsubseteq M_{2}+M_{4}$.

(47) If $M_{1} \sqsubseteq M_{2}$, then $M_{1}+M_{3} \sqsubseteq M_{2}+M_{3}$.

(48) If $M_{1} \sqsubseteq M_{2}$, then $M_{3}-M_{2} \sqsubseteq M_{3}-M_{1}$.

(49) $\quad\left|: M_{1}+M_{2}:\right|$ is less or equal with $\left|: M_{1}:\right|+\left|: M_{2}:\right|$.

(50) If $M_{1}$ is less or equal with $M_{2}$, then $M_{1}-M_{3}$ is less or equal with $M_{2}-M_{3}$.

(51) If $M_{1}-M_{3}$ is less or equal with $M_{2}-M_{3}$, then $M_{1}$ is less or equal with $M_{2}$.

(52) If $M_{1}$ is less or equal with $M_{2}-M_{3}$, then $M_{3}$ is less or equal with $M_{2}-M_{1}$.

(53) If $M_{1}-M_{2}$ is less or equal with $M_{3}$, then $M_{1}-M_{3}$ is less or equal with $M_{2}$.

(54) If $M_{1} \sqsubseteq M_{2}$ and $M_{3}$ is less or equal with $M_{4}$, then $M_{1}-M_{4} \sqsubseteq M_{2}-M_{3}$.

(55) If $M_{1}$ is less or equal with $M_{2}$ and $M_{3} \sqsubseteq M_{4}$, then $M_{1}-M_{4} \sqsubseteq M_{2}-M_{3}$.

(56) If $M_{1}-M_{2}$ is less or equal with $M_{3}-M_{4}$, then $M_{1}-M_{3}$ is less or equal with $M_{2}-M_{4}$.

(57) If $M_{1}-M_{2}$ is less or equal with $M_{3}-M_{4}$, then $M_{4}-M_{2}$ is less or equal with $M_{3}-M_{1}$. 
(58) If $M_{1}-M_{2}$ is less or equal with $M_{3}-M_{4}$, then $M_{4}-M_{3}$ is less or equal with $M_{2}-M_{1}$.

(59) If $M_{1}+M_{2}$ is less or equal with $M_{3}$, then $M_{1}$ is less or equal with $M_{3}-M_{2}$.

(60) If $M_{1}+M_{2}$ is less or equal with $M_{3}+M_{4}$, then $M_{1}-M_{3}$ is less or equal with $M_{4}-M_{2}$.

(61) If $M_{1}+M_{2}$ is less or equal with $M_{3}-M_{4}$, then $M_{1}+M_{4}$ is less or equal with $M_{3}-M_{2}$.

(62) If $M_{1}-M_{2}$ is less or equal with $M_{3}+M_{4}$, then $M_{1}-M_{4}$ is less or equal with $M_{3}+M_{2}$.

(63) If $M_{1}$ is less or equal with $M_{2}$, then $-M_{2}$ is less or equal with $-M_{1}$.

(64) If $M_{1}$ is less or equal with $-M_{2}$, then $M_{2}$ is less or equal with $-M_{1}$.

(65) If $-M_{2}$ is less or equal with $M_{1}$, then $-M_{1}$ is less or equal with $M_{2}$.

(66) If $M_{1}$ is positive, then $M_{2} \sqsubseteq M_{2}+M_{1}$.

(67) If $M_{1}$ is negative, then $M_{1}+M_{2} \sqsubseteq M_{2}$.

(68) If $M_{1}$ is nonnegative, then $M_{2}$ is less or equal with $M_{1}+M_{2}$.

(69) If $M_{1}$ is nonpositive, then $M_{1}+M_{2}$ is less or equal with $M_{2}$.

(70) If $M_{1}$ is nonpositive and $M_{3}$ is less or equal with $M_{2}$, then $M_{3}+M_{1}$ is less or equal with $M_{2}$.

(71) If $M_{1}$ is nonpositive and $M_{3} \sqsubseteq M_{2}$, then $M_{3}+M_{1} \sqsubseteq M_{2}$.

(72) If $M_{1}$ is negative and $M_{3}$ is less or equal with $M_{2}$, then $M_{3}+M_{1} \sqsubseteq M_{2}$.

(73) If $M_{1}$ is nonnegative and $M_{2}$ is less or equal with $M_{3}$, then $M_{2}$ is less or equal with $M_{1}+M_{3}$.

(74) If $M_{1}$ is positive and $M_{2}$ is less or equal with $M_{3}$, then $M_{2} \sqsubseteq M_{1}+M_{3}$.

(75) If $M_{1}$ is nonnegative and $M_{2} \sqsubseteq M_{3}$, then $M_{2} \sqsubseteq M_{1}+M_{3}$.

(76) If $M_{1}$ is nonnegative, then $M_{2}-M_{1}$ is less or equal with $M_{2}$.

(77) If $M_{1}$ is positive, then $M_{2}-M_{1} \sqsubseteq M_{2}$.

(78) If $M_{1}$ is nonpositive, then $M_{2}$ is less or equal with $M_{2}-M_{1}$.

(79) If $M_{1}$ is negative, then $M_{2} \sqsubseteq M_{2}-M_{1}$.

(80) If $M_{1}$ is less or equal with $M_{2}$, then $M_{2}-M_{1}$ is nonnegative.

(81) If $M_{1}$ is nonnegative and $M_{2} \sqsubseteq M_{3}$, then $M_{2}-M_{1} \sqsubseteq M_{3}$.

(82) If $M_{1}$ is nonpositive and $M_{2}$ is less or equal with $M_{3}$, then $M_{2}$ is less or equal with $M_{3}-M_{1}$.

(83) If $M_{1}$ is nonpositive and $M_{2} \sqsubseteq M_{3}$, then $M_{2} \sqsubseteq M_{3}-M_{1}$.

(84) If $M_{1}$ is negative and $M_{2}$ is less or equal with $M_{3}$, then $M_{2} \sqsubseteq M_{3}-M_{1}$.

(85) If $M_{1} \sqsubseteq M_{2}$ and $a>0$, then $a \cdot M_{1} \sqsubseteq a \cdot M_{2}$.

(86) If $M_{1} \sqsubseteq M_{2}$ and $a \geq 0$, then $a \cdot M_{1}$ is less or equal with $a \cdot M_{2}$.

(87) If $M_{1} \sqsubseteq M_{2}$ and $a<0$, then $a \cdot M_{2} \sqsubseteq a \cdot M_{1}$. 
(88) If $M_{1} \sqsubseteq M_{2}$ and $a \leq 0$, then $a \cdot M_{2}$ is less or equal with $a \cdot M_{1}$.

(89) If $M_{1}$ is less or equal with $M_{2}$ and $a \geq 0$, then $a \cdot M_{1}$ is less or equal with $a \cdot M_{2}$.

(90) If $M_{1}$ is less or equal with $M_{2}$ and $a \leq 0$, then $a \cdot M_{2}$ is less or equal with $a \cdot M_{1}$.

(91) If $a \geq 0$ and $a \leq b$ and $M_{1}$ is nonnegative and less or equal with $M_{2}$, then $a \cdot M_{1}$ is less or equal with $b \cdot M_{2}$.

(92) If $a \leq 0$ and $b \leq a$ and $M_{1}$ is nonpositive and $M_{2}$ is less or equal with $M_{1}$, then $a \cdot M_{1}$ is less or equal with $b \cdot M_{2}$.

(93) If $a<0$ and $b \leq a$ and $M_{1}$ is negative and $M_{2} \sqsubseteq M_{1}$, then $a \cdot M_{1} \sqsubseteq b \cdot M_{2}$.

(94) If $a \geq 0$ and $a<b$ and $M_{1}$ is nonnegative and $M_{1} \sqsubseteq M_{2}$, then $a \cdot M_{1} \sqsubseteq$ $b \cdot M_{2}$.

(95) If $a \geq 0$ and $a<b$ and $M_{1}$ is positive and less or equal with $M_{2}$, then $a \cdot M_{1} \sqsubseteq b \cdot M_{2}$.

(96) If $a>0$ and $a \leq b$ and $M_{1}$ is positive and $M_{1} \sqsubseteq M_{2}$, then $a \cdot M_{1} \sqsubseteq b \cdot M_{2}$.

\section{REFERENCES}

[1] Grzegorz Bancerek and Krzysztof Hryniewiecki. Segments of natural numbers and finite sequences. Formalized Mathematics, 1(1):107-114, 1990.

[2] Czesław Byliński. The complex numbers. Formalized Mathematics, 1(3):507-513, 1990.

[3] Czesław Byliński. Some basic properties of sets. Formalized Mathematics, 1(1):47-53, 1990.

[4] Krzysztof Hryniewiecki. Basic properties of real numbers. Formalized Mathematics, $1(\mathbf{1}): 35-40,1990$.

[5] Katarzyna Jankowska. Matrices. Abelian group of matrices. Formalized Mathematics, $2(4): 475-480,1991$.

[6] Eugeniusz Kusak, Wojciech Leończuk, and Michał Muzalewski. Abelian groups, fields and vector spaces. Formalized Mathematics, 1(2):335-342, 1990.

[7] Yatsuka Nakamura, Nobuyuki Tamura, and Wenpai Chang. A theory of matrices of real elements. Formalized Mathematics, 14(1):21-28, 2006.

[8] Andrzej Trybulec. Subsets of complex numbers. To appear in Formalized Mathematics.

[9] Andrzej Trybulec. Tarski Grothendieck set theory. Formalized Mathematics, 1(1):9-11, 1990.

[10] Wojciech A. Trybulec. Groups. Formalized Mathematics, 1(5):821-827, 1990.

[11] Wojciech A. Trybulec. Vectors in real linear space. Formalized Mathematics, 1(2):291296, 1990.

[12] Zinaida Trybulec. Properties of subsets. Formalized Mathematics, 1(1):67-71, 1990.

Received October 19, 2006 